\title{
Analysis of China's Peak Shaving and Natural Gas Storage Systems
}

Natural gas security is one of the core components of energy security, and is an important component part of national security. Experience in many nations has shown that the establishment of a robust natural gas storage and peak shaving system is an effective means to address short-term and mid-term natural gas supply halts and to ensure natural gas industry stable operation as well as stability in the economy and society itself. As China's natural gas demand continues to grow, scaling up this infrastructure in various ways and reasonable deployments of effective emergency-response natural gas reserve systems is the direction and long-term goal of China.

\subsection{Importance of Natural Gas Reserves for Peak Shaving}

As China's economy continues to grow quickly and living standards are rising, as a clean, high-quality and efficient energy, natural gas's share of China's energy consumption is gradually

\footnotetext{
* This chapter was overseen by Zhonghong Wang from the Development Research Center of the State Council and Shangyou Nie from Shell International Exploration and Production. It was jointly completed by Xiaowei Xuan, Yingxie Yang, Yongwei Zhang, Jiaofeng Guo, Weiming Li, Beiqing Yao, Tao Hong and Yuxi Li from the State Resource Department Oil and Gas Centre, Xiaoli Liu from the National Development and Reform Commission's Energy Office, Guang Yang and Jianhong Yang from the China Energy Research Institute's Natural Gas Centre, Xiaobo Ju and Beijing Yao from Shell China. Other members of the topic group participated in discussions and revisions.
}

increasing. Since the official completion in 2004 of the West-East Pipeline project, natural gas consumption has rapidly expanded, and in 2014 it reached 183 billion $\mathrm{m}^{3}$, more than a double the figure in 2004. At the same time, China's natural gas transmission pipeline network is also continually growing and being optimised, with natural gas now among main energies used in urban operations and development and involved in daily lives and urban economic functions. If natural gas supply encounters a shortage or halts, this significantly affects lives and the security of society.

In recent years, China has seen some major shortfalls in natural gas supply in some regions, with winter shortages even leading to a lack of supply for urban residential heating or for taxis wanting to refuel. As a result, ensuring natural gas supply security is of utmost political and economic significance for gas supply enterprises and the cities that use gas. Establishing a natural gas peak shaving reserve emergency response system that corresponds to the scale of city demand is an important undertaking for the sake of China's natural gas supply security.

Systems of natural gas involve upstream production, midstream transmission and downstream utilisation stages, and if any stage exhibits problems, this will affect the stable operation of the entire system. Because downstream users include residents, public services, commerce, industry, power plants, vehicles and air conditioning unit users, various types of user use gas in their own patterns, resulting in fluctuations in gas usage that can result in peaks and troughs in demand. Moreover, the room for production adjustments 
in upstream production is limited, and normally $20 \%$ additional gas volume can be provided at most, making it difficult to satisfy actual peak demand. Therefore, it is necessary to establish peak shaving emergency response facilities to satisfy the peak demand and to deal with sudden gas supply halts.

China's large-scale use of natural gas has only just begun, and natural gas reserve construction is still in its early stages. From the perspective of current development, as China's gas usage grows, the requirements for secure supply will grow. Therefore, it is necessary to integrate China's natural gas market development layout and reserve facility construction, and also to learn from overseas experience in constructing natural gas reserve systems.

\subsection{Issues and Challenges}

\subsubsection{Rapid Increase in Natural Gas Consumption and Peak Period Demand}

\section{China's natural gas demand continues to grow}

China's natural gas consumption growth has been rapid. As China's natural gas resource exploration and development continues to see breakthroughs, proved reserves and production volumes are rising, especially in the Shaanxi-Beijing line, West-East Pipeline and other project locations that are currently involved in commercial operation. China's natural gas industry has entered a stage of rapid growth, and natural gas consumption markets are expanding quickly, with natural gas accounting for an ever-rising proportion of energy consumption structure. In 2014, China's natural gas consumption volume reached 183 billion $\mathrm{m}^{3}$, marking a six-fold increase over 2000, and annual average growth of $15 \%$. The proportion of natural gas among non-renewable energy consumption structure has risen from 2.3 to $6 \%$.

For a significant period into the future, as China's emerging industrialisation and urbanisation accelerates, there will be increasing pressure to improve regional environmental quality and to deal with climate change, actively adjusting energy structures to promote energy conservation and emissions reductions. This is especially true for atmospheric pollution prevention and other environmental protection policies that will be the major policy direction of China in the future, powerfully developing natural gas and other low-carbon energies. This is already a strategic task in the sustainable development of energy in China.

China's natural gas demand will continue to grow rapidly, leading to it becoming one of the major consuming nations of natural gas in the world. The research team believes that in a baseline scenario, natural gas demand in 2015 is likely to approach 200 billion $\mathrm{m}^{3}$, surpass 300 billion $\mathrm{m}^{3}$ in 2020 , exceed 450 billion $\mathrm{m}^{3}$ in 2030, and exceed 600 billion $\mathrm{m}^{3}$ in 2050. In a policy environment of implementation of more active and effective environmental and energy transitioning (such as carrying out carbon trading or collecting a carbon tax), China's demand in 2020 could reach 344.7 billion $\mathrm{m}^{3}$, essentially reaching the goal of 350 billion $\mathrm{m}^{3}$ noted in the 12th Five-Year Plan. By 2030, consumption volume would be likely to reach 580 billion $\mathrm{m}^{3}$, and approach 800 billion $\mathrm{m}^{3}$ by 2050 .

\section{Urban fuel grows fast, as does peak period demand}

Since 2000, China's natural gas consumption structure has tended toward diversification, with urban fuel becoming the fastest-growing sector of gas usage. From 2000 to 2013, China's urban fuel natural gas rose from 4.3 to 73.2 billion $\mathrm{m}^{3}$, with the proportion of total consumption volume rising from 17.6 to $40 \%$. Moving forward, with emerging urbanisation and industrialisation requiring more energy support, and at the same time as ecological cultural construction is strengthening efforts in lifestyle improvements and environmental protection, urban gas in China is set to be pushed toward continued rapid growth in four respects in particular: 
- newly added natural gas users, accelerating the use of LPG and artificial coalbed methane as a replacement in residential gas usage;

- changes made by multiple provinces in relation to coal boiler standards for $\mathrm{PM}_{2.5}$, with increased gas use for heating;

- gas used in transportation and other accompanying infrastructure, which will see rapid rises; and

- distributed energy projects that will successively enter production.

The rapid growth in urban fuel has greatly increased natural gas peak demand. In recent years, in order to improve atmospheric environment quality, northern Chinese regions such as Beijing and Tianjin have developed large quantities of natural gas heating users, including large gas power plants, gas boilers and residential wall-installed heaters. Winter season natural gas usage has skyrocketed. Taking Beijing as an example, its heating season (November-March each year) accounts for approximately $76 \%$ of annual gas usage, with winter and summer peak and trough having a ratio of 12.5:1. Currently, eastern Chinese region winter seasons have yet to implement residential heading, but they also exhibit seasonal characteristics, as peak values occur in the hot summer period and there is a peak to trough ratio of 1.6:1. CNPC's West-East Pipeline company has calculated winter peak demand based on northern and eastern Chinese region natural gas usage load curves, and the two regions' seasonal peak demands are respectively 31 and $4 \%$ of annual gas usage volume (Table 10.1).

In the future, as various regions increase usage of gas heating, seasonal peaks and troughs will continue to increase. According to forecast data by CNPC in 2013 for China's various regional natural gas demands, as well as comparative operation of peak shavings in northern and eastern regions in China, it is possible to calculate China's natural gas peak demand potential. By 2020, natural gas seasonal peal demand will reach 45 billion $\mathrm{m}^{3}$, rising to 76 billion $\mathrm{m}^{3}$ in 2030 . In addition, as gas heating and electricity proportions increase, daily peaks and hourly peaks, among a wide range of problems, will also appear, and must be comprehensively resolved.

\subsubsection{Gas Reserve Peak Shaving Capabilities Insufficient}

\section{Great advances in gas reserve facility construction}

Reserve natural gas peak shaving facilities are an important component part of natural gas

Table 10.1 China's natural gas peak demand calculations (100 million $\left.\mathrm{m}^{3}\right)$

\begin{tabular}{|c|c|c|c|c|c|c|}
\hline \multirow[t]{2}{*}{ W } & \multicolumn{3}{|l|}{2020} & \multicolumn{3}{|l|}{2030} \\
\hline & $\begin{array}{l}\text { Natural gas } \\
\text { demand volume }\end{array}$ & $\begin{array}{l}\text { Peak } \\
\text { factor }(\%)\end{array}$ & $\begin{array}{l}\text { Peak gas } \\
\text { volume }\end{array}$ & $\begin{array}{l}\text { Natural gas } \\
\text { demand volume }\end{array}$ & $\begin{array}{l}\text { Peak } \\
\text { factor }(\%)\end{array}$ & $\begin{array}{l}\text { Peak gas } \\
\text { volume }\end{array}$ \\
\hline Circum-Bohai & 630 & 31 & 195 & 1102 & 31 & 342 \\
\hline North-east & 280 & 31 & 87 & 522 & 31 & 162 \\
\hline North-west & 245 & 31 & 76 & 348 & 31 & 108 \\
\hline $\begin{array}{l}\text { Yangtze River } \\
\text { Delta }\end{array}$ & 595 & 4 & 24 & 928 & 4 & 37 \\
\hline South-east coast & 595 & 4 & 24 & 870 & 4 & 35 \\
\hline South China & 385 & 4 & 15 & 870 & 4 & 35 \\
\hline South-west & 455 & 4 & 18 & 638 & 4 & 26 \\
\hline Mid-west & 315 & 4 & 13 & 522 & 4 & 21 \\
\hline Total & 3500 & & 452 & 5800 & & 764 \\
\hline
\end{tabular}

Data source 2020 data from CNPC Planning Institute, 2030 data from study group arrangements 
transmission system projects. They not only ensure stable pipeline supply but are also integral to strategic reserves and commercial cycles. They are important measures to ensuring the secure and stable supply of natural gas.

China's large-scale gas storage construction began in 1999 with the commencement of the Shaanxi-Beijing Pipeline, in order to resolve imbalances in Beijing's seasonal gas usage and to relieve peak demand, with subsequent large power gas reserve groups and northern China gas reserve groups. Later, along with the construction of the West-East Pipeline, construction of the Jinyun and Liuzhuang storage facilities also began, so that West-East Pipeline market users could use gas as expected. From 2013, China's gas reserve construction saw major progress, and existing gas reserve working gas volume capability is 17.3 billion $\mathrm{m}^{3}$, approaching $10 \%$ of natural gas consumption volume. As far as gas reserve capabilities are concerned, there is still a gap to the global average of $11.3 \%$.

According to the national Natural Gas Development 12th Five-Year Plan, in the next 5-10 years China will continue to heavily promote underground gas reserve construction: "accompanying construction of gas reserve peak shaving facilities are required along longdistance pipelines according to local requirements, with reasonable deployments and clear points of focus, implementing staged construction". It is likely that by about 2020 , China's gas reserve total working gas capabilities will exceed 30 billion $\mathrm{m}^{3}$ (Table 10.2).

LNG commercial reserve capabilities have begun to take shape. As a large quantity of LNG receiving capability comes online, LNG commercial storage in the receiving station, transfer state commercial storage and urban peak shaving LNG storage is receiving increasing attention. Currently, China has yet to have a guiding strategy for LNG reserves, and the country's various large first-stage LNG receiving station designs likewise lack commercial storage beyond ensuring basic load supply. However, they can absorb spot LNG at low prices and release at high prices, while at the same time ensuring that supply security in commercial reserves remains a major point of further development. This is especially true for small and medium-sized LNG receiving and transfer centres, which have relatively small annual transfer capabilities, and approval procedures that are relatively simple. If projects and existing large LNG receiving stations could interconnect, this would not involve LNG import approvals, and would be somewhat simpler, and thus it is currently a focus in the industry. Prior to 2013, China only had two projects in operation, Shanghai Wuhaogou and Dongguan Jiufeng, with annual receiving capabilities of 1.5 million tons in total. China now has more than 10 in-construction, reported or planned LNG receiving and transfer centre projects, and there has been active enthusiasm for investment and construction from local energy companies and private corporations.

In addition to LNG receiving station accompanying construction of reserve facilities, urban fuel is also gradually becoming a major force in LNG reserves. China currently has a certain amount of LNG volume in reserve stations in Beijing, Shanghai, Changsha, Wuhan, Xi'an and Chengdu, ranging from several hundred to over $100,000 \mathrm{~m}^{3}$.

China's commercial reserves are in their infancy, but strategic gas reserves have just begun to build, with only Xinjiang Hutubi representing a functioning large-scale underground strategic gas reserve.

\section{Gas usage load region peak shaving capabilities are insufficient}

Even though there has been some progress in gas reserve facility construction, nonetheless current peak capabilities are still markedly insufficient. Because large-capacity reserves such as Hutubi, Xiangguosi, Suqiao and Jinyun have only been built in the past two years and are still in the gas injection stage, peak shaving capabilities are as yet insufficient, and designed working gas volume is hard-pressed to come into full play.

Currently, winter peak shaving primarily relies upon gas reserves, LNG, gas fields and pressure reduction market gas volumes among other joint peak shaving approaches. Gas field adjustments 


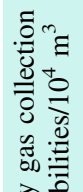

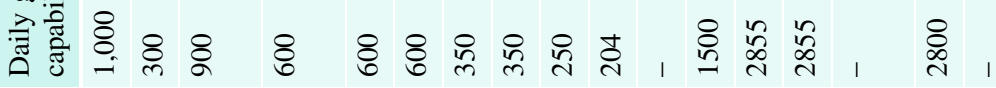

:

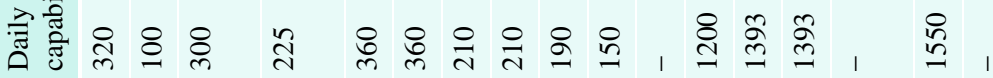

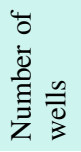

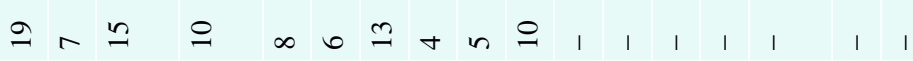

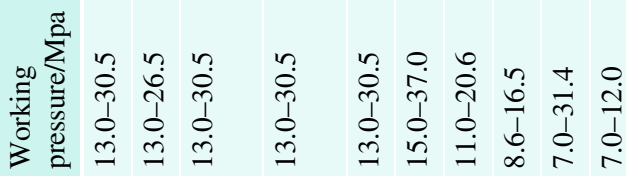

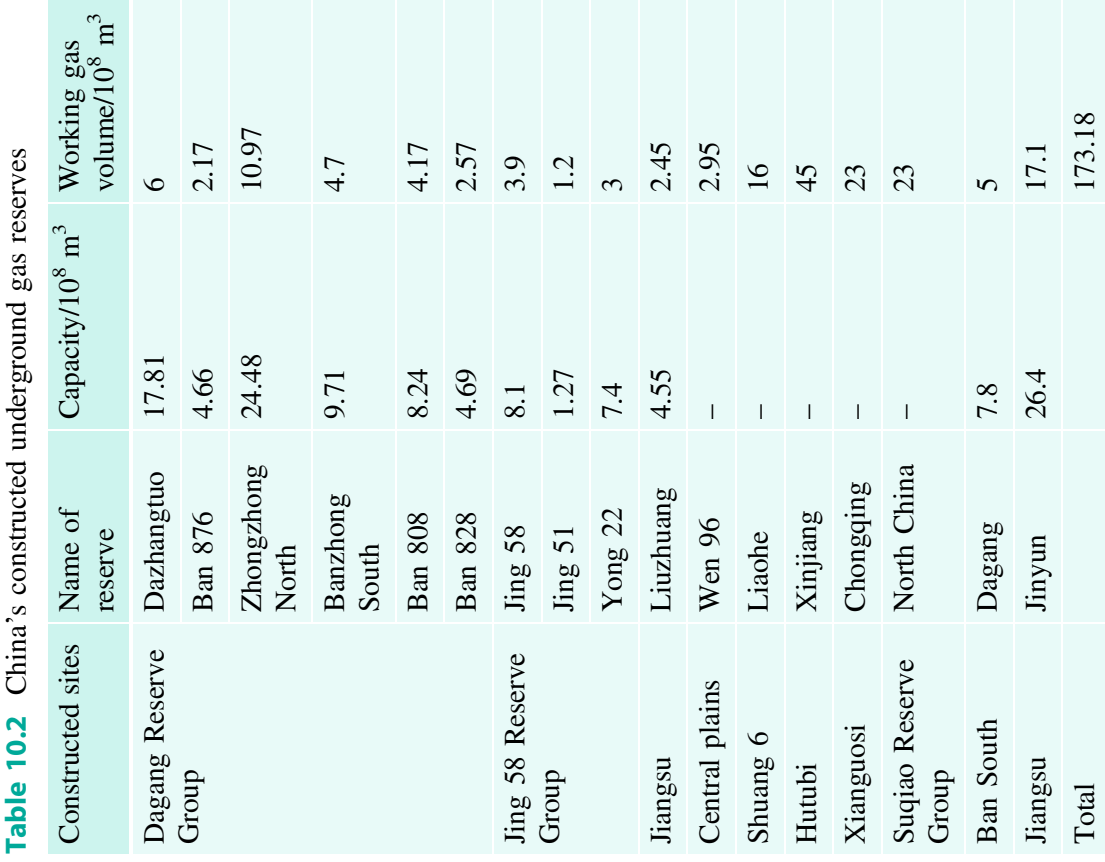


and pressure reduction markets are still the two major modes of peak shaving. For example, in 2012 with its total peak shaving volume of 12.8 billion $\mathrm{m}^{3}$, gas reserve contributions accounted for $16 \%$, LNG for $24 \%$, gas field peak shavings $28 \%$ and pressure reduction markets $31 \%$.

Regions with the largest variance between gas usage peaks and troughs have the most marked shortfalls in peak shaving. The region surrounding the Bohai Sea is the region with China's largest gap between peak and trough, and peak shaving demand is significant. According to forecasts, even if currently constructed and under-construction gas reserves were all successfully established, in 2015 there would still be a shortfall in peak demand of 3.1 billion $\mathrm{m}^{3}$, which will grow to 4.3 billion $\mathrm{m}^{3}$ by 2020 . There are relatively many oil and gas fields around the Bohai region, and there are good conditions for selection of reserve sites. However, there are not many high-quality gas reserve site locations, and the decision can be difficult and the investment requirements high. If the issue of gas peak shaving cannot be resolved, winter peak shaving gas collection capabilities will fall short and major problems could arise.

\section{Pronounced problems with gas reserve prices and operation mechanisms}

Gas reserve facilities are unable to unilaterally create prices, and newly constructed gas reserve facilities are restricted in their development. Currently, China's gas reserves have been supplemented by pipeline facilities and lack independent pricing. Gas reserve stage investments and costs are all linked to pipeline economic efficiency calculations, and corresponding transfer fees are incorporated into pipeline transmission fees, collected together with pipeline transmission fees, and there is no natural gas pricing system to independently establish an item for "gas reserve fees". For example, in 2003 the NDRC announced that the average shipping price (including gas reserve fees) for West-East Pipeline line-wide pipelines was $0.79 \mathrm{CNY} / \mathrm{m}^{3}$.

Prior to the 12th Five-Year Plan, underground gas reserve investments were generally incorporated and collected through pipeline investments as pipeline transmission fees, and gas reserve construction and investment did not have accompanying peak shaving gas prices and transfer fee policies. The nation invested in natural gas commercial reserve facilities, with construction investments (including baseline gas) and 30\% working gas procurement expenses in the form of a rebated income tax that was paid for by the nation, with assets belonging to the group or company and gas reserve and shipping fees assumed by the enterprise. In its guiding opinions regarding acceleration of gas reserve facility construction, the NDRC noted that a reserve price must be established for independently operated gas reserve facilities, but the policy was not implemented.

Gas reserve facility and long-distance pipeline bundled operation affect long-term development. China's natural gas industry is still in the operational mode of having a unified upstream, midstream and downstream. Production, shipping, storage and sale of natural gas is primarily operated and managed by CNPC, Sinopec and other large oil companies. Gas reserves are similar to the early stage of operations in the United States and Europe, and with pipeline auxiliary facilities bundled along with pipelines, there is no independent stage for the natural gas industry chain development. Even though after 2010 there were national investments into construction of gas reserves, nonetheless gas reserve operational models have not undergone fundamental changes. Currently, China's gas reserves are primarily used to co-ordinate supply and demand and peak shavings, as well as for optimisation of production and pipeline network operations, including emergency response and strategic reserves.

\section{Peak shaving and emergency response assurance mechanisms await improvements}

Responsibilities for gas reserve peak shaving are unclear. Currently, China's accountability for natural gas industry chain peak shaving at each stage is unclear, and this causes a divesting of responsibility in emergency natural gas peak shaving within the natural gas industry chain. 
Currently, upstream enterprise peak shaving tasks are excessively challenging, as they not only assume responsibility for regional and seasonal adjustments to gas supply, but also for daily adjustments in key regions. At the same time, China's natural gas emergency response reserve construction investment mechanisms need to be optimised, as they lack the necessary pricing mechanisms and incentive policies. This has led to lack of enthusiasm in the natural gas industry chain upstream, midstream and downstream, causing severe limitations to winter season natural gas peak shaving abilities.

An effective natural gas warning mechanism and emergency response mechanism have yet to be established. Warning forecasts and emergency response mechanisms are important measures for short-term halts of energy supply. Currently, China has yet to optimise mature operating mechanisms in this regard.

In addition, China also lacked progress in matters such as participation in international energy security co-operative frameworks. China has co-operative relationships with essentially all global and regional energy organisations, but not much substantive co-operation, which is not helpful when it comes to using international efforts to co-ordinate dealing with energy security risks. Overseas oil and gas resource co-operations lack effective emergency response mechanisms for sudden incidents, and robust political, foreign relation, economic and even military means are lacking to tackle anti-terrorist emergency response systems and many other plans. As soon as an incident occurs, the nation easily becomes a passive player in events.

\subsection{Key Objectives and Considerations Going Forward}

\subsubsection{Basic Considerations}

Comprehensively implement overall national security requirements, making natural gas supply security an important component part of national security while accelerating deployment of robust and reasonable reserve systems appropriate to the size of the market. As soon as possible, build prompt and flexible warning systems for emergency response, constantly improving China's natural gas supply assurance capabilities, risk avoidance and handling abilities.

\subsubsection{Major Objectives}

Based on the scale of China's future natural gas consumption, current natural gas reserve peak shaving capabilities, underground gas reserve resource conditions and progress in the construction of LNG receiving stations, and with reference to overseas major natural gas-consuming nation experience, this research proposes some 2020 and 2030 objectives for the natural gas commercial and strategic reserve sector in China, as well as emergency response system construction objectives.

In 2020, establish underground gas reserves and LNG reserve integrated peak shaving systems with underground reserves of approximately 50 billion, and LNG reserves of 5-10 billion for a total gas facility working volume of 35-40 billion. This will account for up to $10-11 \%$ of total natural gas consumption volume, changing the current peak shaving method, which is primarily focused on pressure to force reduction of user demand. At the same time, establish an emergency response mechanism for China's natural gas as soon as possible, optimising special project emergency response preparation plans, establishing warning systems for emergency response and ensuring key region gas supply security.

In 2030, further expand the scale and capabilities of the reserve peak shaving system, with gas facility working volume to reach 65 billion and account for $12 \%$ of natural gas consumption volume at that time, reaching global levels. With regard to 2030 natural gas import scale, strategic reserves should reach approximately 5\% of import volume. In keeping with large-scale and full coverage for national natural gas market demands, form effective emergency response reserve systems for fluctuations in natural gas supply and demand. 


\subsection{Recommendations for Developing Natural Reserves for Peak Shaving}

\subsubsection{Accelerate the Formulation of Natural Gas Peak Shaving Emergency Response Plans}

In recent years, some regions in China have encountered frequent gas shortages during the winter season, clearly exposing China's lack of development in natural gas peak shaving reserve facilities, compared to the rapid development of the natural gas market as a whole. In order to guarantee natural gas supply security and the secure operation of the economy, accelerated construction should be pursued by the nation and provincial and municipal governments as well as enterprises to jointly participate in the construction of a top-down natural gas peak shaving emergency reserve system and management system and mechanisms.

It is recommended that national natural gas authorities accelerate the drafting of natural gas peak shaving emergency response reserve plans, making good use of local and enterprise enthusiasm for involvement, ensuring the scale of reserve objectives, the number of emergency reserve days, and deployment and location selection while also incorporating the mid- to long-term natural gas planning of the national and provincial 13th Five-Year Plan. Some underground gas reserve and LNG reserve construction projects meeting certain conditions should be incorporated into the overall framework for national natural gas reserves.

\subsubsection{Emphasise Gas Reserve Facility Legal and Regulatory Construction}

Optimise as quickly as possible the nation's corresponding policies and legislation, ensuring that natural gas reserves have good external policy environments from a systemic perspective so as to encourage enterprises to construct reserve facilities, and carry out research on innovative mechanisms for the reserve operation model. Based on foreign experience, formulate China's natural gas reserve management statutes and reserve laws, clarifying reserve organisation and management institutions and their accountabilities and obligations. For example, in the United States' Natural Gas Act (1983), it was prescribed that the FPC regulates interstate natural gas pipelines, while each state government is responsible for overseeing state natural gas pipelines. China's natural gas authorities should oversee the formulation of plans and policies and carry out regulation of gas reserve facility third-party access, gas reserve prices, reserve usage, emergency mobilisation and corporate operational actions. Enterprise operations and user consumption should be lawfully operated within reasonable frameworks of law and regulation, avoiding abuse of market strengths and promoting effective competition within the market.

Use national laws to clarify the requirements for each level of government, upstream enterprise and fuel enterprise and their responsibilities and obligations in the natural gas reserve system. Implement stepped reserve management systems. The nation is responsible for the strategic reserves of natural gas. Natural gas upstream enterprises assume responsibilities and obligations for seasonable peak shaving and emergency response reserves. Reserve facilities are invested, built and operated by those enterprises, and each province and city's gas enterprises assume daily and hourly peak responsibilities and obligations, with the necessary peak shaving gas reserves being invested in, constructed and operated by those enterprises.

\subsubsection{Accelerate Natural Gas Peak Shaving Emergency Reserve Facility Construction}

The first task is to accelerate construction of large-scale gas reserves and LNG storage tanks 
that can satisfy seasonal peak shaving demands. In northern China, the north-east and the north-west, seasonal peaks and troughs are significant, and there are ample gas reserve locations for resources. First, underground gas reserves should be established, supplemented by LNG, small and medium liquefaction facilities and LNG receiving stations for seasonal peak shaving systems. In central China and the south-west, among other regions with good geographical conditions and nearby oil production areas, use exhausted oil and gas deposits to build underground gas reserves while at the same time using upstream gas fields, with a supplement from small and medium-sized liquefaction facilities. In eastern China, southern China and other locations with relatively poor environments for underground reserves, construction of LNG reserve storage should be a focus, supplemented by underground gas reserves and small and medium-sized storage for peak shaving systems.

The second focus is to construct small peak shaving facilities that can satisfy daily peak demand for gas. In central load gas-using cities, give full play to city fuel companies, accelerating construction of small LNG reserves, CNG tanks and accessory gas reserve facilities to resolve city daily peak shaving demands.

The third focus is to construct natural gas emergency response reserve systems. Clarify natural gas upstream production enterprise and city fuel company emergency reserve responsibilities. Rely upon and expand large reserves of gas, LNG storage and small tank reserves among other facility construction scope, accelerating the establishment of natural gas commercial reserves and satisfying issues of regional supply halt gas demand.

The fourth issue to address is to study the strategy for establishing suitably scaled natural gas reserves. From a global perspective, natural gas has not experienced a supply crisis on a global scale such as the oil crisis, and natural gas security issues are not yet pronounced. However, compared to oil, natural gas has a high degree of unification in the upstream, midstream and downstream. As part of this, if any stage has problems, it will affect the entire chain of natural gas supply security, and there are major potential risks. The 2009 dispute between Russia and Ukraine is one such example of the danger. Therefore, China should develop research into natural gas strategic reserves as quickly as possible upon the basis of properly handling commercial reserves, so as to determine the layout in the mid- to long term for natural gas security issues, making plans early and establishing natural gas strategic reserve scale and models suited to China.

\subsubsection{Formulate Proactive Tax and Price Policies}

Establish reasonable gas reserve pricing mechanisms. The pricing stage in gas reserves must ensure that gas reserve investment and operation costs are recovered while guaranteeing that gas reserve enterprises obtain a reasonable return. On the other hand, standard service and fair competition must be promoted among gas reserve enterprises. The United States has developed peak period/off-peak period or seasonal gas reserve pricing based on service cost pricing approaches that has to a certain degree reduced the risk of imbalance in gas reserve services. In order to promote gas reserve service competition, the United States has also developed market demand pricing approaches. These pricing approaches have improved services to make them more suited to gas reserves, ensuring that gas reserve service providers obtain reasonable economic rewards. China should formulate pricing mechanisms suited to the current state of development of the natural gas industry at the gas reserve stage. Based on the operating costs for gas reserves/storage facilities, and based on government-determined internal rates of return calculations, the government can determine price levels and for each interval of time carry out an assessment and adjustment on gas reserve prices.

Commence differential pricing based on peak and off-peak periods, expressing natural gas value during peak periods. It is recommended that China implement differential pricing for various users during peak and off-peak periods, 
thereby guiding reasonable consumption, achieving a minimisation of peak and off-peak limits and improving system operation efficiency. The peak and off-peak pricing difference in the United States and France is generally 1.2-1.5 fold, determining January-February and November-December as the peak periods for gas usage. By implementing peak gas usage prices, price levels rise from base amounts.

Formulate discount policies and measures to encourage enterprises to construct reserve facilities. Because the construction expenditure for emergency reserve facilities can be enormous, along with major costs for the procurement of reserves and daily operating expenses, and because the main function is to satisfy seasonal peak shaving supply demand, it is recommended that the government put in place specific preferential policies for construction expenses related to facilities for peak shaving reserves. This could, for example, be similar to the approach taken with commercial product oil reserves, with upstream suppliers enabled to use $30 \%$ of LNG reserve costs to construct LNG peak shaving emergency response equipment as a rebate in payable income taxes. This is aimed at encouraging upstream suppliers to construct suitable amounts of LNG reserves so as to deal with temporary supply cuts or extra volume during peak demand periods.

\subsubsection{Accelerate Gas Reserve Management System Reforms}

System reforms include business unbundling, independent settlement and promotion of state-owned oil company gas reserve model reforms. Currently, the United States and European nation gas reserve operations have developed into entirely free-market independent business models, but the model must be within a competitive market environment, including for natural gas supply, shipment and storage stages, which must have many market participants who respect market rules and form a market environment with fair competition.
Considering the current circumstances of China's natural gas industry, future gas reserve operations could first employ a business model without full market liberalisation by establishing a gas reserve operating company within CNPC that is financially independent from the pipeline transmission business, thereby becoming an independent stage of the industry chain. As an independent profit-making entity, the gas reserves company can help facilitate the professionalisation of the gas reserve business and liberalise the market, while also helping to facilitate individual pricing at the gas reserve stage. As China's gas reserve business rapidly grows, it will be partitioned from the pipeline transmission business to become a stage of the industry chain that operates and develops independently.

Gas reserve peak shaving facility construction and operation diversification should be encouraged. Establish a diverse portfolio of entities as part of a gas reserve system, including state-owned oil companies, urban fuel companies and independently operated gas reserve companies. This will be beneficial in ensuring that natural gas supply security and gas reserve capabilities can rapidly grow. Looking at the American experience, this achieved natural gas reserve peak shaving commercialisation to a very high degree, with management and operation entities including interstate and intrastate pipeline companies, local distribution companies and independent gas reserve service providers. In order to encourage private enterprise and other social capital to enter gas reserve facility construction and operation, it is necessary to innovate in a commercial model, forming reasonable price mechanisms that achieve gas reserve facility third-party access and other measures for joint realisation.

\subsubsection{Establish Prompt and Flexible Warning Systems for Emergency Response}

Prompt and flexible warning systems for emergency response means active precautions and 
effective measures to relieve natural gas security risks. The aim is gradually to establish and optimise precautionary emergency response legal systems, organisational institutions and strategic mechanisms, information collection analysis and distribution systems, and various types and levels of emergency preparation and international assistance and co-operation agreement for natural gas emergency response systems.

\section{Establish a natural gas forecast warning system}

Use research to find favourable opportunities to build an indicator system for energy consumption, monitoring systems and evaluation systems to further standardise natural gas statistical systems. Establish statistical data as quickly as possible to monitor and assess as well as to distribute information, wholly integrating energy information channels to continually optimise energy statistics and information collection systems. Enhance forecasting and warning method research and develop forecast warning models suited to China's energy circumstances. Organise a professional team with strong capabilities to establish a timely and flexible forecast warning platform.

\section{Establish an emergency response system covering natural gas production, transmission, sales and all other stages}

Based on gas industry and regional characteristics, determine various levels of emergency response preparation. Establish emergency warning systems in the upstream and midstream focused on the central government and central government enterprises, and downstream plans focused on local governments and local fuel companies. Make full use of the co-ordinating role of the National Energy Council to establish handling for major energy incidents, to provide feedback and for information release review regimes as well as strategy mechanisms, ensuring that emergency response is prompt, strict and authoritative.

\section{Promote communication and co-operation}

China's natural gas forecast warning system construction should likewise be a broad international co-operation. First, strengthen government interdepartmental communication and contact. Strengthen the dialogue and co-operation between the National Energy Administration, National Bureau of Statistics, NDRC Operations Bureau and other relevant departments in the realms of mobilisation, data sharing, forecasting and warning, and other matters so as to establish a unified and well-prepared natural gas forecast warning and emergency response system.

At the same time, in terms of reserve project construction deployment, the National Energy Administration and other local governments should take a scientific approach to planning, with full proofs provided. For approved and authorised project construction sites, various levels of government should offer support to enterprises. In terms of peak shaving emergency response interregional deployment, the National Energy Administration and provincial energy bureaux as well as relevant enterprises should co-ordinate efforts.

Also, strengthen international dialogue and communication relating to energy. In terms of data and information, continue to strengthen data-sharing mechanisms with institutions such as the IEA. Promote dialogue with natural gas source nations including Russia, central Asia, Australia and Qatar, negotiating resolutions to major and outstanding problems. In terms of management experience, make full use of advanced international experience, with management systems and mechanisms to become more scientific in their handling. In terms of research results, fully absorb the research results from various nations and with relation to international organisations, so as to design forecast warning systems suited to China's specific circumstances. 
Open Access This chapter is licensed under the terms of the Creative Commons Attribution 4.0 International License (http://creativecommons.org/licenses/by/4.0/), which permits use, sharing, adaptation, distribution and reproduction in any medium or format, as long as you give appropriate credit to the original author(s) and the source, provide a link to the Creative Commons license and indicate if changes were made.
The images or other third party material in this chapter are included in the chapter's Creative Commons license, unless indicated otherwise in a credit line to the material. If material is not included in the chapter's Creative Commons license and your intended use is not permitted by statutory regulation or exceeds the permitted use, you will need to obtain permission directly from the copyright holder. 